Please share your stories about how Open Access to this article benefits you.

\title{
Utilization of Time-Based Techniques in Research and Teaching
}

by Paola Sanguinetti

2006

This is the published version of the article, made available with the permission of the publisher. The original published version can be found at the link below.

Sanguinetti, Paola. (2006). Utilization of Time-Based Techniques in Research and Teaching. International Journal of Architectural Computing 3(4):63-77.

Published version: http://www.dx.doi. org/10.126o/147807706778658775

Terms of Use: http://www2.ku.edu/ scholar/docs/license.shtml 


\section{Utilization of Time-Based Techniques in Research and Teaching}

Paola Sanguinetti

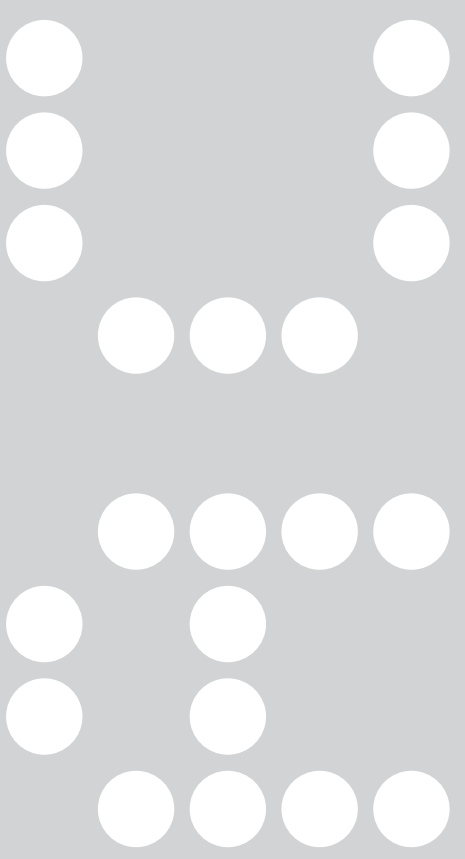




\section{Utilization of Time-Based Techniques in Research and Teaching}

Paola Sanguinetti

This article describes how computer animations, combined with cinematic techniques, have served as a teaching and research tool in architecture. Animations were used in the following areas: case studies of precedents in architecture using the animated modeling as a form of tectonic analysis; conceptualization of space through the study of film, specifically three approaches using film in the early stages of design: cinematic structure, film as site, and transcoding transparency; and mapping the urban fabric combining various time-based media. 


\section{INTRODUCTION}

The use of the computer has affected the modes of production of architects and the experience of architecture. In Terminal Velocities, Stan Allen proposes areas of investigation that transcend the arguments for or against computers in the design studio and critically look at this tool's capabilities and constraints. "Specifically this would imply understanding the computer not in utopian terms (turning away from matter and reality), but in more pragmatic terms: articulating a more complex interplay of the real and the virtual."' In Towards a Paperless Studio, Frederick Norman points out that digital media can be a tool of investigation in the early stages of design and examines the issues that arise in providing a knowledge base. "If students are not adequately taught the digital design skills at the appropriate level in their education, digital media will not become part of a process of design and upper level studios will be degraded to instructional labs for software training." $\quad$ Throughout my teaching of digital studios, I have emphasized a combination of hands-on techniques with digital media. I believe that within the notion of studio as a forum for synthesis, a synthesis of modes of representation is required. I have found that when a project begins with a physical model and then a digital model, the level of detail of the digital model will be greater, and the use of animation enhances the level of development of the digital model. In the scope of this paper, the focus is on the specific use of computer animations used extensively as a technique to merge research, analysis, and design. Most software packages used in architectural modeling include in their interface the camera view. With the use of the virtual camera, architects are able to set up multiple views to simultaneously build and explore space, and to create walk-through or flythrough animations, which are in essence short movies of a project. This is to me the most radical difference between making a digital model versus a physical one: the ability to experience the space of the model in time which is essential to the experience of architecture. This has led me to the study of film to inform the use of the virtual camera when producing animations. In teaching computer modeling in design studios and elective courses, I have developed 3 approaches to teaching and using computer animations combined with cinematic techniques: I) case studies of precedents in architecture using the animated exploded modeling as a form of tectonic analysis; 2) conceptualization of space through the study of film, specifically three approaches using film in the early stages of design: cinematic structure, film as site, and transcoding transparency ${ }^{3}$; 3) mapping the urban fabric combining various time-based media: animated abstract and representational models, edited still images, and video.

\section{TECTONIC ANALYSIS THROUGH ANIMATED MODELING}

The following two animation techniques were developed as tools for 
analysis and case studies of precedents in architecture, first as part of my own research, and then as a teaching tool in 3 elective courses. I conducted a study of two private houses designed by two leading Latin American architects: Oscar Niemeyer, known for the curvilinear white forms in the city of Brasilia, and Lina BoBardi, best known for her Modern Art Museum in Sao Paolo - a cube made of concrete and glass suspended on four red concrete piers. I used modeling as a tool for comparative analysis of their work and I focused on the private residences that each architect designed as their private home in the same time period of the 1950's. My aim was to produce an accurate model of these two houses, and in so doing, gain an understanding of the tectonic composition as an expression of each architect's design ideas. The choice of the modeling software 3dStudio Max to conduct this study allowed for the critical study of form, material, texture, and light. This work led to a grant to create a digital archive of digital models of Latin American Architecture. In addition to allowing for the study of architecture in this region from my own perspective as a Latin American, the goal of this archive was to make the knowledge of these architectural works accessible to students at the University of Kansas. The archive was produced through a series of electives courses. The idea for the course was to combine the development of computer skills with the acquisition of knowledge of a specific historical time period of Latin American Architecture. The course comprised of workshops on rendering, material, lighting, interactive software, followed by student presentations. Each student applied the newly acquired computer skills to construct a digital model of a built work in Latin America. Students were asked to make an analysis of the selected building through the use of animations. In order to construct a digital model, each student utilized plans, sections, and photographic documentation. The specific stages of the course included a) the application of modeling techniques in the building analysis through the construction of a white model; b) the animation of exploded axonometric of the white model; c) applying material and lighting in order to test the model against the real through the production cinematic fade animations; $d$ ) and the production of additional walk-though animations, and compilation all animations in the form an interactive presentation for of each case study.

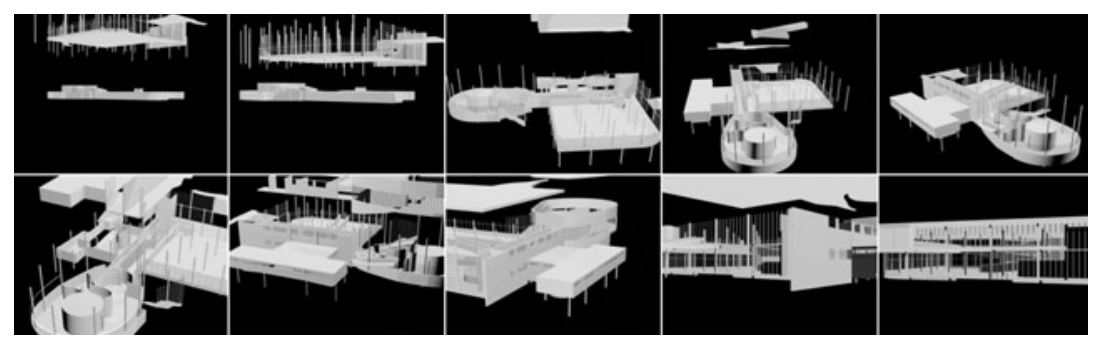

\section{I Exploded Axonometric Animation}

The idea for this type of animation (figure I) came from the use of the
Figure I: Still images of the exploded axonometric animation of the Niemeyer's Pampulha Casino 
exploded axonometric drawing as a tool of representation used by architect to show the components of a project. In Architectural Graphics, Frank Ching describes two types of single view drawings, the perspective view and the paraline or axonometric view. He further describes the expanded view, also known as exploded axonometric, as a method of analysis: "A building form can also be expanded along the $x-y-z$ axes of a paraline drawing to illustrate how its construction components relate to one another and/or to selectively reveal the interior spaces within." 4 The exploded axonometric view appears as a suspended moment in an animation where, the pieces of the building have been pulled apart ready to reassemble or continue expanding following a path of motion parallel to the Cartesian axes. This view can be achieved in the computer by rotating the axis in an orthogonal view, also called user view. In terms of the animation, it requires building the model with the intention to animate its assemblage. It therefore requires a clear understanding of the architects design intent as well as the methods of construction or building assemblage. For the purpose of the digital archive, students began to understand the selected building by looking closely at the drawings and other available material, in order to model each component with the intention to show them coming together, assembling the building. Students were asked to model the building in white for two reasons. Keeping a monochromatic scheme maintained the focus in the analytical reading and the animation of the building as a tectonic assemblage. This also enabled the students to learn about materials and lighting in two stages, first applying procedural maps to develop texture and transparency as basic parameters of a material, and then in the next set of animations using bitmaps, color, and lighting, to gain an understanding of parameters such as specular highlight, and the relationship and of materials and lighting.

\subsection{Cinematic Fade}

A fade is a cinematic editing technique where one image dissolves into another. In films it is used to connect or to separate two scenes depending on the length of the fade or if one scene dissolves into another or fades to black (or white). I first used this technique in my own animations of Casa Canoas by Oscar Niemeyer, and Casa de Vidro, by Lina BoBardi. I used the fade to show the connection between the digital model and the real building. By placing the virtual camera in order to generate a view that matched the photographs of the building, my aim was to show how that the virtual and the real fit exactly. I then created an animation where the path of the camera linked all those locations and faded in the photographic image. This was achieve through a rigorous process of a) setting up the virtual camera to match the exact location and angle of the real camera for each photograph, and b) to connect all the camera positions as a continuous sequence to be animated, $c$ ) to create the fades using video editing software and the digitized photographs. Matching a virtual camera with a photograph can be done by bringing the photograph into the viewport and matching it 
visually or through a technique called camera matching. This requires locating points in the camera view and inputting their coordinates. To do this with multiple images can be a very painstaking process... In the case of the elective courses dedicated to the study of Latin American architecture, the students produced distinct separate animations for each fade sequence, as the overall animation became long and complicated for the students.

These animation techniques were also used in a subsequent study of the work of Antonio Gaudi and Santiago Calatrava, which originated from the interest in understanding the link between these two Spanish architects. The common theme between the two is the extensive use of abstracted natural forms in the design and development the building's structural systems and the use of the arch as structural element. I continue to implement the use of the exploded axonometric animation in my digital studios as part of a one-week research project. This is an opportunity for students to conduct precedent research, learn basic modeling and animation techniques, and acquire a basic knowledge for the subsequent design work in the digital studio.

\section{THE INTEGRATION OF CINEMA IN THE DIGITAL DESIGN STUDIO}

The three methodologies described here were used in $4^{\text {th }}$-year architecture studios over five semesters. The study of film was introduced for a variety of reasons: the school of architecture had introduced the option of "digital or paperless studios" where students were required to own and use computers. Most digital studios implemented the use of AutoCAD to expedite the production of architectural drawings, where the computer was used for drafting and the production of 2-dimensional drawings. Some studios used 3d Studio Max to produce final renderings and presentation images of the models produced with CAD. I wanted to tap the potential of this software during the design process. In exploring the connection between film and architecture, my goal was to integrate the use of the virtual camera, in the preliminary stages of design, as a device to frame and simulate the experience of moving through space. The projects begin with the analysis of film following three steps: scene selection, analysis, and physical and digital modeling to synthesize ideas. I chose relatively recent films not only to engage the students' interests, but also to provide a relevant depiction of the contemporary urban experience to students coming from other backgrounds, such as small towns or suburban areas in the Midwestern United States. The idea was to extract a conceptual model of space from the study of film and to use it in the development of architectural space. I believe that this could be done only through animations because of the possibility of immersion into the digital model enabling the architect to transform a concept model into an architectural model. 
Figure 2: Example of a physical model and still images from the cinematic structure animation

\section{I Cinematic structure}

In this approach, the class studied the ways in which different directors constructed space with the camera. The visualization and modeling techniques used to analyze film were then to be used in the development of the architectural design, and the digital model produced in this exercise was the source of design concepts to be integrated with the program of the building. Contemporary films were selected specifically because of their depiction of our contemporary experience through the manipulation of time and space within the conventions of cinematic narrative, conveying ideas such as globalization, sprawl, and time compression. Each student was to select a film, from a short list provided, and then a scene. To understand the structure of the scene, first the sequence of the scene was broken down into a series of film stills. The analysis was done via diagrams to document camera angles, aperture, framing, montage, composition, focus, etc. The analysis of each film still helped establish a connection between the movie camera and the space that recorded in the scene. After the structure of the scene was dissected, physical models were used to translate the analysis into 3-dimensional material. The physical models also aided in the construction of the digital models. Once in the digital realm, students used the virtual camera to inhabit the spaces of the model, effectively mimicking the angles, aperture, and movement of the camera in the film sequence. As an example, one student selected a scene in Being John Malchovich where the viewer's plane of reference is continuously changed. As the camera moves through walls and ceilings, the walls of the previous room turn into the floor or ceiling of the next room. His study model was a bass wood structure of stationary opaque planes and movable translucent panels. The physical model was designed to have no base, no top or bottom. The animation of the computer model choreographed the movement of the virtual camera with the sliding of the panels, and the roll camera with the rotation of the digital model (Figure 2). The design of the model was revised for the path of motion of the virtual camera, by relocating the position of the stationary pieces, or changing the sliding or pivoting of the translucent panels.

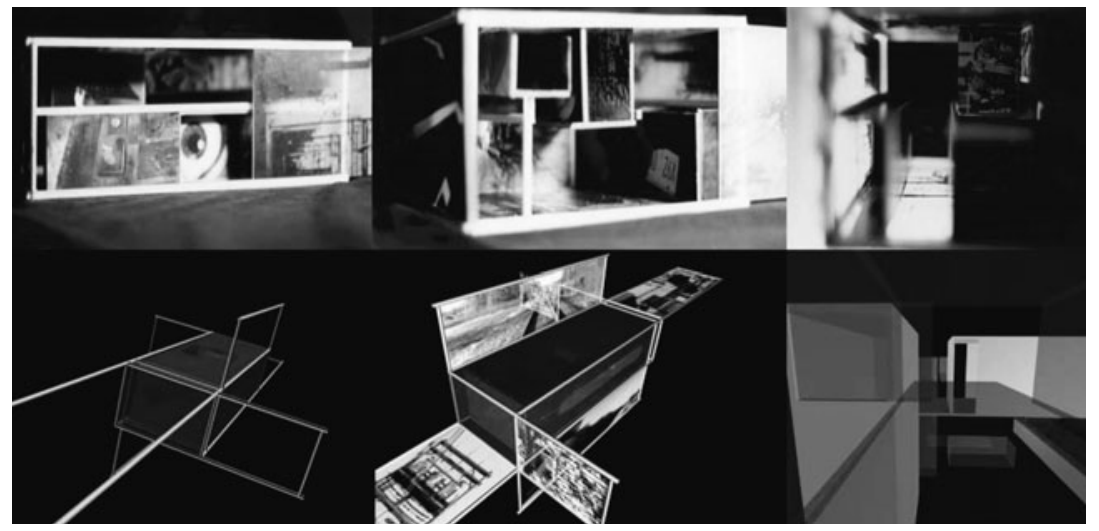

Utilization of Time-Based Techniques in Research and Teaching 


\subsection{Film as site}

In this approach, cinematic immersion supplemented site analysis in two different projects:ACADIA's student competition for the Berlin Inforum, and the master plan for the World Trade Center site in New York. Film was a way to visit places quite remote from our location in Lawrence, Kansas.

In the case of Berlin, students watched Wings of Desire by Wim Wenders and Run Lola Run by Tom Tyker, and were asked to observe the cinematic techniques to describe the city. In Wings of Desire, the city is fragmented, divided by the wall. This boundary is expressed by juxtaposing two temporal experiences in the same space: the chromatic time of the living with the grayscale timelessness of the angels. In Run Lola Run, the urban experience is non-linear. Two spaces are compressed in the cinematic frame and time is stretched and looped. Students were asked: (I) to select a scene from each film to establish a comparative analysis of Berlin, (2) to translate the ideas generated from the analysis into physical concept-model, and (3) to produce a digital model and animation. The models produced 3dimensional maps and surfaces representing the spatial and temporal qualities of the city in their texture, and other material properties. The sense of speed depicted in Run Lola Run was represented in the animations in two ways, in the application of materials with elongated specular highlights or raytraced reflections, and in the editing techniques using blurring effects. Digital morphing techniques showed the evolution and transformation of Berlin's urban morphology with the destruction of the Berlin wall.

The digital models and the rendered animations became sources of ideas for the students' design proposals. This occurred as the students developed, used, and transformed the formal compositions of their digital models. It also happened as students appropriated the experiences captured by the virtual camera in the animations. The results were an emphasis on the materiality and surface properties of the Inforum, both as the articulation of the building's skin as well as a media and information surface.

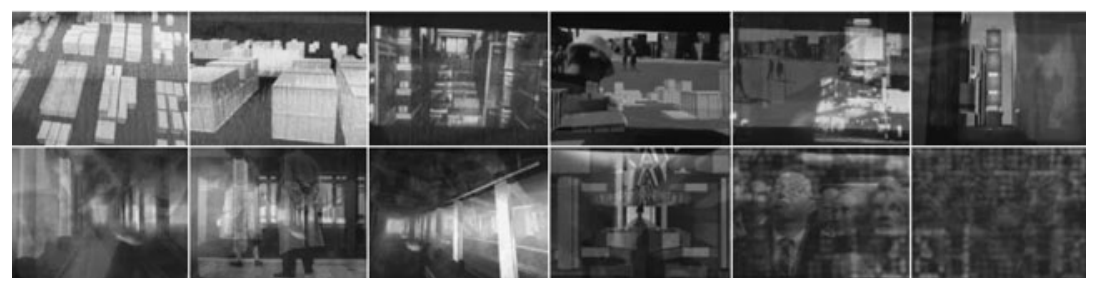

Figure 3: Still images of the New York Documentary Animation

In the case of the site analysis for a master plan for the World Trade Center, students were asked to look at films of New York to get a sense of the city beyond the images connected to September II. Because none of my students had been to New York, I thought there was a risk that their work might simply become a planimetric organization of functions on a site 
that had been presented to them by the Media. Before addressing the complexities of the programmatic brief provided by the Lower Manhattan Development Corporation, students were asked to assemble a documentary of New York (figure 3). They combined sequences captured from film and animations produced from their own digital model of Manhattan. Here the virtual camera was a tool to explore the spaces created by abstracting the city's morphology into a digital model. One group of students selected Wall Street, Oliver Stone's depiction of the city in the late 80 's. The corrupted materialistic system was expressed in the film as a physical entity, in the verticality of the city and the urban grid of tall buildings with an array of generic offices and expensive apartments. The group made computer models of specific urban locations such as the subway stop, Times Square, and the trading floor of the New York Stock Exchange. To represent the relationship between the reduction of the individual to a commodity and the compression and globalization of space, the students' animation showed the fluctuations of scale and proportion between these urban spaces layered with the density of noises and people.

These two approaches used film to provide the direct experience of site. The students appropriated the filmmaker's vision and translated it into the spatial vocabulary of the architect, as seen in the resulting computer models and animations.

\subsection{Transcoding transparency}

The third study is a more open-ended approach to film as a medium. The focus is on the experience of transparency as a phenomenon manifested in film at many levels, ranging from physical to psychological, from the condition of looking through reflective glass, to the act of seeing ourselves in dreams.

Students chose a film conveying an experience of transparency, then a scene to be analyzed. They developed physical models to study and map the patterns of light, the movement of the camera, and other cinematic techniques from their chosen scene. This time, the three-dimensional models were produced at full-scale. The physical models, as installations pieces, reintroduced the phenomenology of transparency in material terms while also engaging our bodies (Figure 4). These installations were in essence screens, abstracting the composition of the cinematic frames. The screens also revealed other aspects of transparency, from the fluctuations of moiré patterns in the surface of a stretched veil, to a structure of translucent layers defining a crystalline volume. For Bernard Tschumi, "neither space nor concept alone is erotic, but the junction between the two is" 5 . In this sense, each screen contained and dissolved the perception of space from mental construct into sensual experience. The screen produced worked as devices to distance our bodies from the space beyond, all the while letting us have glimpses of it, and therefore creating a desire to 
be in that space. Here the physical model, the screen, became a threshold into virtual space. Phenomenological reality spliced into virtual reality; by recording the physical effects of the screen such as the translucency of fabric in light or the flickering of metal, and combining them through mapping and editing with the digital model.

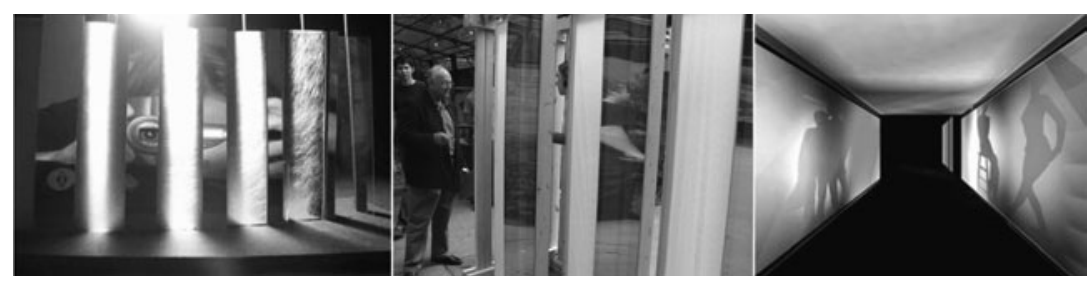

\section{MAPPING THE URBAN FABRIC}

These two projects explore the critical potential of montage, defined by Eisenstein as a space of dialectic uniting architecture with film. ${ }^{6}$ The animations focus on mapping the urban fabric combining various time-based media: animations of abstract and representational models, edited still images, and video. The possibilities of mapping, as a way of understanding, recording, and learning, from the contemporary urban condition, specifically in the United States, were studied in Tschumi's Manhattan Transcripts and Venturi's Learning from Las Vegas. In the project Sampling Kansas City, the technique of montage is used to exploit the juxtapositions of virtual and digital space to make a critique of the city. In the project Mapping New Orleans, animations are edited to bring together mapping and modeling to visualize the future of the city.

\section{I Sampling Kansas City}

In the Manhattan Transcripts, Bernard Tschumi applies storyboarding used in film to the documentation and conceptualization of architectural space. Each of the four episodes of the city is based on a three-part device in which events, spaces, and movements are represented by photographs, architectural drawings, and choreographic notation. This mapping device undergoes variations throughout the series of episodes, and becomes a collection of fragments to convey the urban experience. Following this technique of Tschumi's Manhattan Transcripts, students selected four sites in Kansas City. Starting with the first site, documentation was organized in a triptych of EVENT, SPACE, and MOVEMENT. The class began with by researching two cinematic techniques: the long take and the jump cut and the writings of Eisenstein on montage and Guy Debord's on derive. Using these various cinematic approaches, students used video to capture the direct experience of site. Student video taped the selected spaces several times to document changes in use, light, noise, etc. The video was then edited down to I-minute sequences. The animations were used as graphs
Figure 4: Example of a small scale model, large scale model or screen, and still image from the resulting virtual environment animation 
Figure 5: Still images of the animation mapping of 4 malls in Kansas City

to interpret, analyze, and synthesize this video data. Two types of animations were used to represent space and movement. Animations of digital models of the city such as the exploded axonometric were used to analyze space. Animations of wireframe models and particle systems were used to diagram movement. The methodology evolved as each site was mapped combining the three distinct time-based techniques. For example, a student documenting four malls in Kansas City created an exploded axonometric animation, containing an animated particle system to show the density of users within the mall. He also created a tag to show to his location within the density, connecting it to the superimposed frame of the video while also tracking his movement within the axonometric model (Figure 5). In this project, the study of cinematic techniques provided the basis for the urban analysis, and enabled the students to communicate their ideas in the form of four sequential animations.

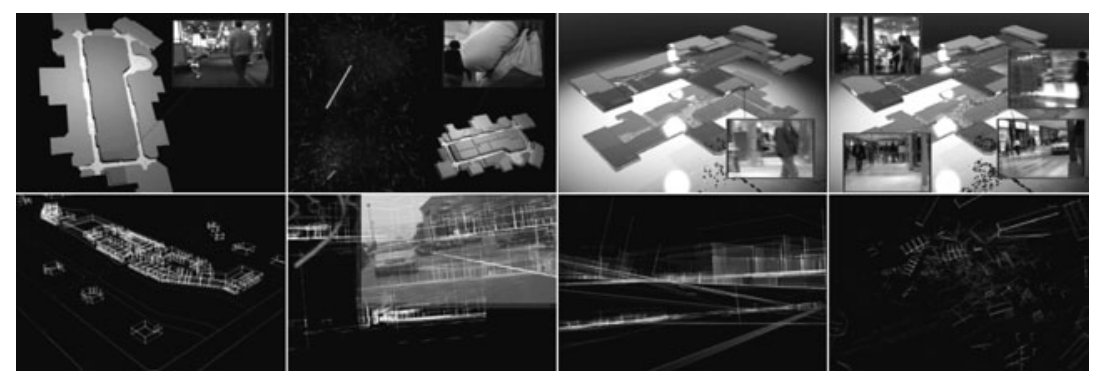

\subsection{Rebuilding New Orleans}

In After the Flood, Michael Sorkin asks for a reconsideration of our roles as architect to rebuild the fabric of New Orleans. "Rebuilding must meditate decisively on sprawl and density, engage the role of renewables in powering our cities and lifestyles, act dramatically to expand public transportation, and revisit planning and building codes for sustainability and self-protection. Decisive, careful, and comprehensive planning is an imperative, the only way to mitigate the effects of outrageous poverty on the fabric and organization of the city, to recast urban organization along lines informed by 2 Istcentury knowledge." 7 This project begins as an analytical mapping of New Orleans, identifying and mapping parameters relevant to rebuilding the urban fabric. Students were asked to collect data to inform their proposals, ranging from maps on the historical development of the city, to aerial imagery of existing conditions. Students were asked to build and animate a series of models. Starting with one overall map of New Orleans, to show their visions for the city, the goals was then to shift scales and modes of representation, and model five specific instances within the urban proposal to examine site conditions, programmatic combinations, space and morphology. Here the raw data for analysis are the various 2-dimensional maps, GIS data, and aerial photographs. The animations become a synthetic 
medium integrate all the data into a system of interrelated components that can be studied in time. The goal was to explore their potential of animation to be tools for analysis as well as tools to convey ideas of space and experience. Beyond the expectation of the animation to be determined by the path of motion of the virtual camera, the animation became a device to reveal aspects of the model. For example, some animations focused on materiality, specifically changing levels of opacity of certain objects from solid to clear in order to reveal internal relationships; other animation explored the morphing and folding of surfaces of the model to explore the idea of growth and evolution of the urban fabric over time. The animations enable the student to abstract the city in animated system.

In essence, this final approach to animations is a result of the knowledge acquired in my previous exercises. In both projects, subject of study is the contemporary urban condition, using animations to convey the dynamic relationship between the physical and non-physical aspect of the city. Animation enables the exploration of ideas in time. The animations are structured as a series of vignettes ${ }^{8}$, as animated fragments of the urban experience. Cinematic editing techniques connect the various scales and fragments.

Eisenstein argued that "Montage is conflict" where new ideas emerge from the collision of images and where the new emerging ideas are not innate in any of the images of the edited sequence. In the juxtaposition of scenes, a new concept explodes into being.

\section{DISCUSSION}

The projects and approaches described in this article chronicle an evolution in the use of animation in computer electives and digital studios at the University of Kansas. In the projects discussed in this paper, students acquired technical skills ranging from the understanding of the digital interface, the use of the virtual camera, the animation of parametric models, and video-editing to produce cinematic effects. Computer animations provided students with the ability to study the space of the model and its parameters in time. Animations combined with the application cinematic techniques enabled them to record and convey the temporal condition of architecture.

In the first approach animations were used for case studies of precedents in architecture in various computer elective courses. The exploded axonometric animation was a transformation of a known representational tool, the axonometric drawing, and the animated fade, served as a way to test the accuracy of the digital model to the real, similar to using a scale to measure a physical model or a drawing.

The second approach introduced animations in the studio in the preliminary stage of design. Film and cinematic techniques were the focus of study to begin the process to generate design concepts in the form of 
animations. Three topics were studied. a) Cinematography and sequence. With the knowledge acquired from the analysis of cinematic structure, students produced complex models and animations to explore various ideas associated with our perception of time and space, such as compression, blurring, folding, etc. b) The movement of the camera and the actors, and the mise-en-scene. By looking at film as site, students were able to understand qualitative aspects of a city they had not visited, and developed a concept of place, albeit mediated by the director's cinematic vision. c) Narrativity and editing. ${ }^{9}$ In this study of the phenomenology of transparency in film, students experimented with the connection between the construction of the real and the virtual and explored aesthetic and material aspects of transparency. In the case of the projects conducted in the digital studios using animation and film, physical models were used to facilitate the transition between one kind of virtual space into another through tectonic means. The results ranged from explorations of space and forms that sometimes can only be built in the digital realm, to expressions of material qualities beyond the traditional model-building materials used in architecture.

The third approach to the use of animations was a direct result of the knowledge acquired in the previous two approaches. Animations of various digital models were combined with video and digital images to map the urban condition. The cinematic technique of montage remained the compositional and editing tool to assemble and structure various animated sequences.

It is important to note that these 3 approaches had the same goal: to accelerate the learning of computer modeling and the integration of digital media in the toolkit of architecture students. These approaches were developed over time and are discussed here in the order in which they were developed. Animations and cinematic editing techniques were first used as an analytical tool in the study of existing buildings, revealing their tectonic composition and establishing a connection to the real. Then animations were the medium to explore the connection between architecture and film and the experience of space, site, and materiality. Finally animations were used both to convey the analysis and the experience of urban space and its transformation over time. In these three approaches, the study of film and the use of cinematic techniques were important because like film, animations have a narrative structure.

\section{CONCLUSION}

Animations are currently being used in schools and offices, but mainly for presentation. Parallels have been made before between the filmmaker and the architect, which are more relevant now that through the use of modeling software we can produce animations. With the virtual camera architects are able to understand space in four dimensions. Once immersed, 
the visualization of the mind can be explored in virtual space to create virtual environments and to experience material, scale, and light, as we design, construct the digital model. Through the use of animations, the virtual spaces produced in these projects illustrate a layered condition. William Mitchell describes this condition in City of Bits, where he proposes that our direct physical experience not only has the same value as the images mediated through film, television, and the Internet, but also that the urban environment and therefore our concept of public space are being restructured by the code of the computer. ${ }^{10}$ The results of these projects suggest that the study of film in the conceptual phase of design expands our understanding of contemporary culture. As a result, the architectural project, like film, could be conceived as a story, an animation mediated through computation, and simultaneously layering digital information with cultural meaning. The next area of research in the use of time-based techniques will be related to the idea of immersion into the space of the digital models. Animations like film exist with in the structure of the narrative, where the viewer is passive. There are other forms of immersion into digital space where the user has multiple choices such as surfing the web or playing video games. In many aspects video games can me similar to films, but here the user is given control of the virtual camera. This radical difference, the idea of interactivity, opens up possibilities for architecture. I believe the study of video games, both the evolution in the graphic depiction of space and the interactive structure of games, are a logical continuation of this study.

\section{Acknowledgements}

Part of this work was possible through two Research Grants from the School of Architecture and Urban Design at the University of Kansas.

\section{References}

I Allen, S., Terminal Velocities:The Computer in the Design Studio, in:Allen, S., Practice: Architecture, Technique, and Representation, G+B Arts International, Australia, 2000, $145-160$

2 Norman, f., Towards a Paperless Studio, in: Proceedings of ARCC Spring Research Conference, 2001, 85-91 http://www.asu.edu/caed/arcc/articles/NORMAN.PDF [30-04-2006]

3 Manovich, L., The Language of New Media, MIT Press, Cambridge, 2002, 45

${ }^{4}$ Ching, F., Architectural Graphics, Van Nostrand Reinhold Inc., New York, I 985, 60

5 Tschumi, B., Questions of Space, AA publications, London, 1990.

${ }^{6}$ Eisenstein, S., Montage and architecture, Assemblage 10, MIT Press, Cambridge, Mass., 1989, III-I3I

7 Sorkin, M., After the Flood: Rebuilding the Physical and Social Fabric, Architectural Record.com, 2005 http://archrecord.construction.com/news/katrina/critique.asp [30-04-2006]

8 http://en.wikipedia.org/wiki/Vignettes [30-04-2006] 
${ }^{9}$ http://en.wikipedia.org/wiki/Narrativity[30-04-2006]

10 Mitchell,W., City of Bits, MIT Press, Cambridge, Mass., 1995

Paola Sanguinetti

University of Kansas

School of Architecture and Urban Design

I 465 Jayhawk Boulevard, Lawrence, KS 66046

paolas@ku.edu 
